OPEN ACCESS

Edited by:

Valentina Tozzini,

Nanosciences Institute, National

Research Council, Italy

Reviewed by:

Sebastien Fiorucci,

University of Nice Sophia

Antipolis, France

Matteo Tiberti,

Danish Cancer Society Research

Centre (DCRC), Denmark

*Correspondence:

Josef Melc

j.melcr@rug.n

Jean-Philip Piquemal jean-philip.piquemal@

sorbonne-université.fr

†These authors have contributed equally to this work

Specialty section

This article was submitted to Biological Modeling and Simulation,

a section of the journal

Frontiers in Molecular Biosciences

Received: 09 September 2019 Accepted: 20 November 2019

Published: 04 December 2019

Citation:

Melcr J and Piquemal J-P (2019) Accurate Biomolecular Simulations Account for Electronic Polarization.

Front. Mol. Biosci. 6:143.

doi: 10.3389/fmolb.2019.00143

\section{Accurate Biomolecular Simulations Account for Electronic Polarization}

\author{
Josef Melcr ${ }^{1 * t}$ and Jean-Philip Piquemal ${ }^{2,3,4 *+}$ \\ ${ }^{1}$ Groningen Biomolecular Sciences and Biotechnology Institute and the Zernike Institute for Advanced Materials, University of \\ Groningen, Groningen, Netherlands, ${ }^{2}$ Laboratoire de Chimie Théorique, Sorbonne Université, UMR7616 CNRS, Paris, \\ France, ${ }^{3}$ Institut Universitaire de France, Paris, France, ${ }^{4}$ Department of Biomedical Engineering, The University of Texas at \\ Austin, Austin, TX, United States
}

In this perspective, we discuss where and how accounting for electronic many-body polarization affects the accuracy of classical molecular dynamics simulations of biomolecules. While the effects of electronic polarization are highly pronounced for molecules with an opposite total charge, they are also non-negligible for interactions with overall neutral molecules. For instance, neglecting these effects in important biomolecules like amino acids and phospholipids affects the structure of proteins and membranes having a large impact on interpreting experimental data as well as building coarse grained models. With the combined advances in theory, algorithms and computational power it is currently realistic to perform simulations with explicit polarizable dipoles on systems with relevant sizes and complexity. Alternatively, the effects of electronic polarization can also be included at zero additional computational cost compared to standard fixed-charge force fields using the electronic continuum correction, as was recently demonstrated for several classes of biomolecules. Keywords: molecular dynamics simulations, electronic polarization, electronic continuum correction,
biomolecules, phospholipids, amino acids, nucleic acids, ions

In molecular dynamics simulations, the interactions between molecules are described with approximate potentials known as force fields that mimic the true Born-Oppenheimer energy hypersurface. Among these methods, pairwise additive potentials are very popular for modeling biomolecules such as proteins, lipids or nucleic acids (Ponder and Case, 2003; Lopes et al., 2015). The current standard force fields (Huang and MacKerell, 2013; Maier et al., 2015; Robertson et al., 2015), however, neglect important physical many-body effects such as the electronic polarization, charge transfer, or many-body dispersion (cited in decaying magnitude order) (Kleshchonok and Tkatchenko, 2018). Although such models have provided valuable insight into many phenomena from various fields including biology, chemistry, biophysics, or material sciences, there are several important cases in which accounting for polarizability is crucial.

\section{PITFALLS OF NON-POLARIZABLE FORCE FIELDS}

The limited predictive accuracy of non-polarizable force fields led the molecular modeling community to develop new generation "polarizable" force fields (Gresh et al., 2007; Jorgensen, 2007; Stone, 2013; Shi et al., 2015; Piquemal and Jordan, 2017; Kleshchonok and Tkatchenko, 2018; Martinek et al., 2018; Melcr et al., 2018, 2019; Antila et al., 2019; Jing et al., 2019) able to include the missing physics with a special focus on the polarizability effects. Although such techniques are now widely used in fields studying highly charged ionic liquids (Bedrov et al., 2019), their application 
cannot be limited only to such extreme cases. For instance, neglecting the effects of the electronic polarizability in important biomolecules like amino acids, nucleic acids, and phospholipids affects the structure of proteins (Jiao et al., 2008; Shi et al., 2009; Duboué-Dijon et al., 2018a), DNA (Babin et al., 2006), and membranes (Harder et al., 2009; Catte et al., 2016; Melcr et al., 2018) having a large impact on interpreting experimental data (Hauser et al., 1977; Eisenberg et al., 1979; Kurland et al., 1979; Feigenson, 1986; Mattai et al., 1989; Roux and Bloom, 1990, 1991; Böckmann and Grubmüller, 2004; Lund et al., 2008; Vacha et al., 2009; Berkowitz et al., 2012; Melcrová et al., 2016; Javanainen et al., 2017; Magarkar et al., 2017) as well as building coarse grained models. Importantly, these results show that the electronic polarization yields non-negligible effects also at overall neutral molecules (Gresh et al., 2007; Melcr et al., 2018).

Secondary structure of proteins is to a large extent determined by an intricate network of hydrogen bonds. The description of hydrogen bonds in standard force fields, however, does not contain important contributions, e.g., from polarization and partially covalent character (Babin et al., 2006). It was demonstrated in many cases including structure of water (Dang, 1998), binding of ligands to proteins (Friesner, 2005; Jiao et al., 2008), and protein folding and unfolding (Morozov et al., 2006; Freddolino et al., 2010; Piana et al., 2011, 2014; Huang and MacKerell, 2014; Lemkul et al., 2016; Célerse et al., 2019) that polarizability contributes significantly to the accuracy of simulations of structures with hydrogen bonds. Also, salt bridging between amino acids is likely overestimated in strength when the effects of polarization are not included (Friesner, 2005; Vazdar et al., 2013; Debiec et al., 2014; Ahmed et al., 2018; Célerse et al., 2019; Mason et al., 2019). For instance, the interaction of acidic side chains of glutamate and aspartate with cations is overestimated in strength in classical non-polarizable force fields (Patel et al., 2009; Duboué-Dijon et al., 2018a), while treatment of polarizability in solvent relaxation affects salt bridge dissociation (Célerse et al., 2019). Taken together, the secondary and tertiary structural arrangements in the simulations of proteins are likely biased to certain preferred configurations due to the lack of polarizability depending on the chosen parametrization strategy (Freddolino et al., 2009, 2010; Piana et al., 2011, 2014).

Membrane proteins form a large part of cellular proteome and are in direct contact with amphiphilic cellular membranes, which influence their structure and activity (Lee, 2004). Membranes themselves are crucial cell organelles which define the inner resp. outer cellular environment. They are predominantly composed of amphiphilic phospholipids, which self-assemble into stable bilayer structures (Harayama and Riezman, 2018). The force fields for phospholipids have been tuned to the level that the simulations of commonly used simplified model lipid membranes can reproduce a large variety of experimentally measured properties, phenomena and structural features including lipid self-diffusion, x-ray scattering patterns, bilayer thickness, area per phospholipid, and acyl chain order parameters (Pluhackova et al., 2016).

This could make an impression that the currently available non-polarizable lipid force fields provide comparable accuracy to the models with explicit polarization at a fraction of the computational cost. While the non-polarizable models yield accurate results in many cases (Lucas et al., 2012; Chowdhary et al., 2013a), simulation studies have revealed that such models gradually lose their predictive accuracy with increasing complexity beyond model systems used during their parametrization, e.g., when membranes are put into contact with buffers of physiological ionic strengths (Catte et al., 2016). For instance, improvements in the electrostatics of phospholipid membranes have a great impact on the membrane dipole potential, permeation of water through membranes, and viscosity of organic liquids (Harder et al., 2009; Venable et al., 2019). Moreover, the interactions between phospholipids and cations, especially divalent cations like calcium, are overestimated in the classical non-polarizable models (Catte et al., 2016; Melcr et al., 2018; Antila et al., 2019).

In general, the structure of divalent cations complexes that are widespread in biosystems is traditionally problematic in non-polarizable simulations (Kohagen et al., 2015). In contrast, simulations with explicit or implicit treatment of polarization yield comparable accuracy to DFT-based ab-initio calculations and neutron scattering experiments, as was demonstrated for biologically relevant divalent cations $\mathrm{Ca}^{2+}$ and $\mathrm{Mg}^{2+}$ (Piquemal et al., 2006b; Wu et al., 2010; Martinek et al., 2018). While accounting for the electronic polarization overall improves the predictive accuracy of simulations in general, it is not sufficient in some cases like zinc chloride ion pairing, where more complex physics beyond "mere" electronic polarization is at play (Gresh et al., 2005, 2007; Piquemal et al., 2007; Duboué-Dijon et al., 2018b).

\section{IMPLICIT TREATMENT OF ELECTRONIC POLARIZATION VIA ELECTRONIC CONTINUUM CORRECTION}

The necessity of polarizability and screening in modeling lipid bilayers has been an issue from the very beginning of computational modeling of model membranes. The first pioneering works on phospholipid bilayers document the need of including polarizability and extra screening in the development of the first models, which was achieved at that time through an empirical scaling factor for the partial atomic charges of the phospholipids (Egberts et al., 1994). A similar strategy supported by continuum theory was used in the recent developments of phospholipid force fields, which implicitly account for the electronic polarization using Electronic continuum correction (ECC) (Leontyev and Stuchebrukhov, 2009, 2010a; Mason et al., 2012; Pegado et al., 2012; Pluhařová et al., 2013; Martinek et al., 2018). Despite the approximate treatment of the polarizability using ECC, such lipid force fields provide accurate interactions between phospholipid bilayers and cations in agreement with experiments (Melcr et al., 2018). In particular, in the case of the neutral phosphatidylcholine (PC), ECC improved the cation binding affinity for monovalent, and divalent cations reaching agreement with experiments (Melcr et al., 2018), while for negatively charged phosphatidylserine (PS) it has also improved 
the overall structure of the phospholipid and the interactions with other lipids (Antila et al., 2019; Melcr et al., 2019).

Electronic continuum correction is a very efficient alternative to otherwise computationally demanding explicit modeling of electronic polarization (Bedrov et al., 2019). The accuracy of the ECC method was shown to yield promising results on several polar organic solvents (Leontyev and Stuchebrukhov, 2010b, 2012; Lee and Park, 2011; Vazdar et al., 2013), while it proved to be necessary yet sufficient for an accurate description of the structure of several monovalent and divalent ions in aqueous solutions (Mason et al., 2012; Pegado et al., 2012; Pluhařová et al., 2013). To date, the array of force fields utilizing ECC has grown from a wide range of biologically relevant ions (Kohagen et al., 2014, 2015; Martinek et al., 2018), to protein moieties (Vazdar et al., 2013; Duboué-Dijon et al., 2018a; Mason et al., 2019), and whole phospholipid molecules (Melcr et al., 2018) making realistic simulations of e.g., membrane proteins at physiological ionic conditions possible.

In ECC all particles are assumed to have equal polarizabilities and the electric field and electron density within each particle is homogenous (Leontyev and Stuchebrukhov, 2009). Such approximations simplify the calculations of the polarization to such an extent that it can be simply included in the interactions as a pre-determined charge-scaling factor (Leontyev and Stuchebrukhov, 2009), which is derived from the highfrequency dielectric constant of electrons, $\varepsilon_{e l}$, as $1 / \sqrt{\varepsilon_{e l}} \approx 0.75$ for aqueous solutions. Importantly, $\varepsilon_{e l}$ is close to 2 for a wide variety of biologically relevant environments meaning that even interfaces like biological membranes do not give rise to large gradients. Despite the coarseness of the approximations, the effects of electronic polarization are described sufficiently well for a variety of biologically relevant molecules in a condensed phase (Duboué-Dijon et al., 2018a,b; Martinek et al., 2018; Melcr et al., 2018). Moreover, ECC accounts for the effects of electronic polarization at zero additional computational cost compared to standard fixed-charge force fields. Although, a new generation of simulation codes performing large scale simulations with explicit polarization models starts to emerge (Lagardère et al., 2018), ECC yields the benefit of employing the widely adopted and already highly optimized codes for classical MD.

The common implementation of ECC via charge rescaling profoundly resembles an empirical scaling factor, which, obviously, reduces the interaction of charged molecules. From both the derived ECC theory (Leontyev and Stuchebrukhov, 2010a) and its applications, which compare ECC to also other methods (Pegado et al., 2012; Martinek et al., 2018), it is however clear that the improvements pertinent to ECC can be attributed to the electronic polarization. For instance, interactions of sulfate anions were directly compared between simulations with ECC, solvent shell model (Rick and Stuart, 2003) and ab-initio calculations (Pegado et al., 2012). This comparison has revealed that ECC performed comparably well to the other methods at a fraction of the computational cost. Moreover, ECC was concluded as preferable over the explicit solvent shell model for sulfate anions as it was closer to the structures from ab-initio calculations (Pegado et al., 2012).

\section{CAPTURING EFFECTS BEYOND ELECTRONIC POLARIZATION}

The accuracy of the implicit methods including ECC is limited and gradually becomes inadequate in cases, which do not adhere to the assumed approximations. For instance, the complex electronic structure of $\mathrm{Zn}^{2+}$ makes it difficult to capture the ion pairing of zinc chloride with ECC unless specific ad hoc interaction terms between the ions are introduced (Duboué-Dijon et al., 2018b). Hence, resorting to more accurate modeling strategies including explicit polarizable dipoles-or even effects beyond electronic polarization-becomes necessary in such cases.

The AMOEBA force field with explicit polarizable dipoles correctly reproduces water structure around $\mathrm{Zn}^{2+}$ in bulk solution and its free energy of hydration, however, it still does not capture the fine details of zinc chloride ion pairing. The reason for that is that $\mathrm{Zn}^{2+}$ exhibits considerably large charge transfer effects prefiguring what is happening with transition metals where back-donation effects become important (Gresh et al., 2005, 2007; Piquemal et al., 2007). Simulations then need to utilize more complex polarizable force fields able to separately evaluate the different physical contributions. Indeed, short-range electrostatics in such systems is anything but classical as it is strongly affected by quantum penetration effects in the overlap region (Piquemal et al., 2003, 2006a; Gresh et al., 2005, 2007; Wang et al., 2015). On the contrary, many-body polarization interactions which are usually cooperative (i.e., the total energy being larger that the purely additive contributions) do not behave in such a way (Gresh et al., 2007, 2016; Zhang et al., 2012; Jing et al., 2018). Divalent metal cations in particular locally reverse the physical trends and exhibit net anticooperativity as the total energy becomes smaller than the sum of individual contributions. For example SIBFA (Sum of Interactions Between Fragments $\mathrm{Ab}$ initio computed) incorporates a many-body explicit charge transfer (Gresh et al., 2005, 2007; Piquemal et al., 2007) and a penetration correction for electrostatics (Piquemal et al., 2003; Narth et al., 2016), and is able to deal with such difficult systems.

Such effects also exist with variable magnitude in biomolecular simulations, and resorting to more accurate methods employing physics even beyond explicit polarization will be likely required for predictive accuracy in many cases, e.g., metalloproteins, which shall be interesting playgrounds for such modeling (Gresh et al., 2007, 2016; Zhang et al., 2012; Jing et al., 2018). Improvements in capturing correct physics is a general trend in current developments, and besides SIBFA, the AMOEBA force field is gradually evolving into the AMOEBA+ potential, which additionally includes such physical effects (Liu et al., 2019). Moreover, several other general polarizable potentials are emerging (Huang et al., 2017; Das et al., 2019; Rackers and 
Ponder, 2019) indicating the start of next-generation polarizable force fields development (Piquemal et al., 2006a; Duke et al., 2014; Piquemal and Cisneros, 2016).

\section{ARE POLARIZABLE SIMULATIONS COMPUTATIONALLY TRACTABLE?}

This being said the question remains: is there any practically achievable perspective application of such advanced models to meaningfully large simulations of biologically relevant systems?-Certainly yes. If the use of polarizable models has been doomed by their computational cost for years, things have dramatically improved. In terms of computational requirements, the approaches utilizing Drude particles (Lopes et al., 2013) traditionally appeared more feasible compared to explicit point dipole approaches (Lipparini et al., 2014; Lagardère et al., 2015), as their computational cost in standard high-performance codes was higher by a factor 2-4 depending on implementation and reference settings compared to non-polarizable force fields (Jiang et al., 2011), while the explicit point dipoles models were roughly twice slower. However, such models cannot utilize long time-steps because of their use of extended Lagrangian, which practically imposes a speed limit (Wang and Skeel, 2005; Albaugh and Head-Gordon, 2017). In contrast, utilizing advanced algorithms for solving polarization and dynamical integration is possible within explicit point dipole approaches leading to strong speed increases to the performance level of Drude approach (even for higher-level multipolar electrostatics approaches such as AMOEBA) when compared to usual non-polarizable models simulation (Lagardère et al., 2019). However, the numerous available point dipole force fields (AMOEBA, SIBFA etc...) had in practice another handicap besides their computational cost: they were not available in high performance/production codes such as GROMACS or NAMD (Phillips et al., 2002; Van Der Spoel et al., 2005).

This situation has gradually changed in recent years. First, in link with the improved multi-timestep integration, the key mathematical problem of solving the point dipole equations using iterative methods was alleviated using new non-iterative approaches such as the Truncated Conjugate Gradient (TCG1) (Aviat et al., 2017a,b) that allows for a fix cost evaluation of polarization. When coupled to an analytical evaluation of gradient such an approach fully preserves energy and, hence, allows for long time step simulations. Second, the availability of massively parallel MPI codes able to efficiently use supercomputers using $3 \mathrm{D}$ domain decomposition techniques such as Tinker-HP (Lagardère et al., 2018; Jolly et al., 2019) [the high performance engine of the Tinker molecular package Rackers et al., 2018] shed first rays of light at the end of the tunnel leading toward simulations of biologically relevant large systems on long enough timescales with explicit polarization. Moreover, GPU accelerated implementations of AMOEBA in OpenMM (Huang et al., 2018) and Tinker-OpenMM (Harger et al., 2017) are available whereas the support of hybrid (multi)CPUs-GPUs is coming in Tinker-HP (O. Adjoua et al., personal communication).
Overall, methodology has made a key progress and will continue in this direction for all types of polarizable force fields as the accessible computer power quickly increases reducing therefore the computational gap with additive potentials. Whereas specialized highly accurate water potentials based on many-body expansions emerge such as MBPOL (Riera et al., 2019) and allow for a better understanding of fine physical effects in clusters and bulk water, the availability of general polarizable force fields such as AMOEBA offering water (Ren and Ponder, 2003), ions, organochlorine compounds ( $\mathrm{Mu}$ et al., 2014), proteins and nucleic acids (Shi et al., 2013; Zhang et al., 2018) now enables performing enough sampling to achieve highly accurate and biologically meaningful simulations. The Drude approaches parametrization is expanding as well (Lamoureux et al., 2003; Chowdhary et al., 2013a,b; Lopes et al., 2013). Moreover, accelerated sampling methods start to be applied also to polarizable approaches (Célerse et al., 2019) offering improved simulation capabilities and access to accurate and fast evaluation of free energies of binding thanks to GPUs (Harger et al., 2017). Such capabilities allow to tackle hard systems as in the case of the Phosphate binding mode of the Phosphate-binding protein where it was possible to highlight the critical effect of the buffer solution ending a long standing controversy thanks to free energy computations (Qi et al., 2018).

\section{SUMMARY: BIOMOLECULAR SIMULATIONS OF THE FUTURE ARE POLARIZABLE}

In summary, we have presented several important classes and case studies of biomolecules, where including polarizability is an important factor for the simulation accuracy. Cytosolic environment in cells is mostly composed of water solutions of ions, for which polarizability is necessary for the accurate description of the solvated structure of ions, their pairing and interaction with other biomolecules (Piquemal et al., 2006b; Wu et al., 2010; Mason et al., 2012; Pegado et al., 2012; Pluhařová et al., 2013; Duboué-Dijon et al., 2018a,b; Martinek et al., 2018; Melcr et al., 2018). Polarizability is an important factor for accurate interactions between amino acids, namely salt bridges between them, which are overestimated in strength in current non-polarizable force fields (Friesner, 2005; Vazdar et al., 2013; Ahmed et al., 2018; Célerse et al., 2019; Mason et al., 2019). Moreover, polarizable force fields yield a better description of the hydrophobic effect and hydrogen bond networks in proteins, which to a large extent determine the dynamic structure and conformational changes of proteins (Dill et al., 1995; GarcíaMoreno et al., 1997; Fitch et al., 2002; Morozov et al., 2006; Freddolino et al., 2010; Piana et al., 2011, 2014; Huang and MacKerell, 2014; Lemkul et al., 2016; Célerse et al., 2019; Venable et al., 2019). Polarizability is necessary for accurate structure and interactions of both neutral and charged phospholipids, which constitute a dominant part of cellular membranes (Harder et al., 2009; Catte et al., 2016; Melcr et al., 2018).

The representation of electronic polarization in classical MD simulations can vary largely with Drude and induced point 
dipoles approaches on one side and continuum approximations on the other (Cieplak et al., 2009; Lopes et al., 2009; Leontyev and Stuchebrukhov, 2011; Schröder, 2012; Baker, 2015; Shi et al., 2015; Lemkul et al., 2016; Bedrov et al., 2019; Jing et al., 2019). With the advances in both computational power together with theory and algorithms it is practically achievable to perform simulations with explicit polarizable dipoles on systems with relevant sizes and complexity (Qi et al., 2018; Bedrov et al., 2019; Lagardère et al., 2019; Loco et al., 2019). In particular, it is currently realistic to perform simulations with explicit polarization at time scales, which are competitive to the standard fixed-charge simulations (Lemkul et al., 2016; Lagardère et al., 2018; Célerse et al., 2019). Moreover, advanced polarizable potentials (e.g., SIBFA, AMOEBA+) including effects even beyond electronic polarization are being actively developed to tackle systems with complex structure like metalloproteins, kinases or ribozymes (Gresh et al., 2007, 2016; Zhang et al., 2012; Jing et al., 2018, 2019; Das et al., 2019; Liu et al., 2019; Rackers and Ponder, 2019). Also, approximate implicit solutions like ECC, which circumvent the computational costs of explicit polarization, gradually gain on popularity and provide a promising solution for a variety of applications in biomolecular simulations (Duboué-Dijon et al., 2018a,b; Martinek et al., 2018; Melcr et al., 2018, 2019; Mason et al., 2019). Finally, as fully variational polarizable embeddings are now possible in hybrid QM/MM molecular simulations (Loco et al., 2016, 2017, 2019), one can expect that hybrid explicit polarization/ECC simulations will be possible in the near future offering a multilevel global treatment of polarization across very large complex molecular systems.

Biomolecules in the real world cannot turn off their polarizability. Hence, molecular dynamics simulations, which aim to give a realistic, robust, and predictive results, cannot afford to neglect this important contribution to the electrostatic

\section{REFERENCES}

Ahmed, M. C., Papaleo, E., and Lindorff-Larsen, K. (2018). How well do force fields capture the strength of salt bridges in proteins? PeerJ 6:e4967. doi: $10.7717 /$ peerj. 4967

Albaugh, A., and Head-Gordon, T. (2017). A new method for treating drude polarization in classical molecular simulation. J. Chem. Theory Comput. 13, 5207-5216. doi: 10.1021/acs.jctc.7b00838

Antila, H., Buslaev, P., Favela-Rosales, F., Ferreira, T. M., Gushchin, I., Javanainen, M., et al. (2019). Headgroup Structure and Cation Binding in Phosphatidylserine Lipid Bilayers. J. Phys. Chem. B. 123, 9066-9079. doi: 10.1021/acs.jpcb.9b06091

Aviat, F., Lagardère, L., and Piquemal, J.-P. (2017a). The truncated conjugate gradient (TCG), a non-iterative/fixed-cost strategy for computing polarization in molecular dynamics: Fast evaluation of analytical forces. J. Chem. Phys. 147:161724. doi: 10.1063/1.4985911

Aviat, F., Levitt, A., Stamm, B., Maday, Y., Ren, P., Ponder, J. W., et al. (2017b). Truncated conjugate gradient: an optimal strategy for the analytical evaluation of the many-body polarization energy and forces in molecular simulations. J. Chem. Theory Comput. 13, 180-190. doi: 10.1021/acs.jctc.6b00981

Babin, V., Baucom, J., Darden, T. A., and Sagui, C. (2006). Molecular dynamics simulations of DNA with polarizable force fields: convergence of an ideal B-DNA structure to the crystallographic structure. J. Phys. Chem. B 110, 11571-11581. doi: 10.1021/jp061421r interaction. Currently, polarizable force fields for a large variety of biomolecules and simulation codes implementing polarizability exist and are readily available to solve various biophysical problems (Wu et al., 2010; Chowdhary et al., 2013a; Lemkul et al., 2016; Duboué-Dijon et al., 2018a,b; Lagardère et al., 2018; Martinek et al., 2018; Melcr et al., 2018; Zhang et al., 2018; Bedrov et al., 2019; Célerse et al., 2019; Jing et al., 2019; Liu et al., 2019). We expect that the popularity of such approaches will grow and will become a common tool in biomolecular research in the near future.

\section{DATA AVAILABILITY STATEMENT}

All datasets generated for this study are included in the article/supplementary material.

\section{AUTHOR CONTRIBUTIONS}

All authors listed have made a substantial, direct and intellectual contribution to the work, and approved it for publication.

\section{FUNDING}

This work has received funding from the European Research Council (ERC) under the European Union's Horizon 2020 research and innovation programme (grant agreement No. 810367), project EMC2.

\section{ACKNOWLEDGMENTS}

We thank the organizers of the CECAM workshop 2019 Multiscale Modeling from Macromolecules to Cell: Opportunities and Challenges of Biomolecular Simulations for their kind invitation to this special research topic.
Baker, C. M. (2015). Polarizable force fields for molecular dynamics simulations of biomolecules. Wiley Interdiscipl. Rev. 5, 241-254. doi: 10.1002/wcms.1215

Bedrov, D., Piquemal, J.-P., Borodin, O., MacKerell, A. D., Roux, B., and Schröder, C. (2019). Molecular dynamics simulations of ionic liquids and electrolytes using polarizable force fields. Chem. Rev. 199, 7940-7995. doi: 10.1021/acs.chemrev.8b00763

Berkowitz, M. L., Vácha, R., and Vacha, R. (2012). Aqueous solutions at the interface with phospholipid bilayers. Acc. Chem. Res. 45, 74-82. doi: $10.1021 / \operatorname{ar} 200079 \mathrm{x}$

Böckmann, R. A., and Grubmüller, H. (2004). Multistep binding of divalent cations to phospholipid bilayers: a molecular dynamics study. Angew. Chem. Int. Ed. 43, 1021-1024. doi: 10.1002/anie.200352784

Catte, A., Girych, M., Javanainen, M., Loison, C., Melcr, J., Miettinen, M. S., et al. (2016). Molecular electrometer and binding of cations to phospholipid bilayers. Phys. Chem. Chem. Phys. 18, 32560-32569. doi: 10.1039/ $\mathrm{C} 6 \mathrm{CP} 04883 \mathrm{H}$

Célerse, F., Lagardère, L., Derat, É., and Piquemal, J.-P. (2019). massively parallel implementation of steered molecular dynamics in tinker-hp: comparisons of polarizable and non-polarizable simulations of realistic systems. J. Chem. Theory Comput. 15, 3694-3709. doi: 10.1021/acs.jctc.9b00199

Chowdhary, J., Harder, E., Lopes, P. E. M., Huang, L., MacKerell, A. D., and Roux, B. (2013a). A polarizable force field of dipalmitoylphosphatidylcholine based on the classical drude model for molecular dynamics simulations of lipids. J. Phys. Chem. B 117, 9142-9160. doi: 10.1021/jp402860e 
Chowdhary, J., Li, H., Harder, E., Lopes, P., MacKerell, A., and Roux, B. (2013b). Drude model based polarizable force field for lipids. Biophys. J. 104:31a. doi: 10.1016/j.bpj.2012.11.210

Cieplak, P., Dupradeau, F.-Y., Duan, Y., and Wang, J. (2009). Polarization effects in molecular mechanical force fields. J. Phys. Condens. Matter 21:333102. doi: 10.1088/0953-8984/21/33/333102

Dang, L. X. (1998). Importance of polarization effects in modeling the hydrogen bond in water using classical molecular dynamics techniques. J. Phys. Chem. B 102, 620-624. doi: 10.1021/jp9731258

Das, A. K., Urban, L., Leven, I., Loipersberger, M., Aldossary, A., Head-Gordon, M., et al. (2019). Development of an advanced force field for water using variational energy decomposition analysis. J. Chem. Theory Comput. 15, 5001-5013. doi: 10.1021/acs.jctc.9b00478

Debiec, K. T., Gronenborn, A. M., and Chong, L. T. (2014). Evaluating the strength of salt bridges: a comparison of current biomolecular force fields. J. Phys. Chem. B 118, 6561-6569. doi: 10.1021/jp500958r

Dill, K. A., Bromberg, S., Yue, K., Fiebig, K. M., Yee, D. P., Thomas, P. D., et al. (1995). Principles of protein folding-a perspective from simple exact models. Protein Sci. 4, 561-602. doi: 10.1002/pro.5560040401

Duboué-Dijon, E., Delcroix, P., Martinez-Seara, H., Hladílková, J., Coufal, P., and Kř́žzek, T., et al. (2018a). Binding of divalent cations to insulin: capillary electrophoresis and molecular simulations. J. Phys. Chem. B 122, 5640-5648. doi: 10.1021/acs.jpcb.7b12097

Duboué-Dijon, E., Mason, P. E., Fischer, H. E., and Jungwirth, P. (2018b). Hydration and ion pairing in aqueous $\mathrm{Mg} 2$ and $\mathrm{Zn} 2$ solutions: forcefield description aided by neutron scattering experiments and ab initio molecular dynamics simulations. J. Phys. Chem. B 122, 3296-3306. doi: 10.1021/acs.jpcb.7b09612

Duke, R. E., Starovoytov, O. N., Piquemal, J.-P., and Cisneros, G. A. (2014). GEM$^{*}$ : a molecular electronic density-based force field for molecular dynamics simulations. J. Chem. Theory Comput. 10, 1361-1365. doi: 10.1021/ct500050p

Egberts, E., Marrink, S. J., and Berendsen, H. J. C. (1994). Molecular dynamics simulation of a phospholipid membrane. Eur. Biophys. J. 22, 423-436. doi: 10.1007/BF00180163

Eisenberg, M., Gresalfi, T., Riccio, T., and McLaughlin, S. (1979). Adsorption of monovalent cations to bilayer membranes containing negative phospholipids. Biochemistry 18, 5213-5223. doi: 10.1021/bi00590a028

Feigenson, G. W. (1986). On the nature of calcium ion binding between phosphatidylserine lamellae. Biochemistry 25, 5819-5825. doi: 10.1021/bi00367a071

Fitch, C. A., Karp, D. A., Lee, K. K., Stites, W. E., Lattman, E. E., and Bertrand García-Moreno, E. (2002). Experimental pKa values of buried residues: analysis with continuum methods and role of water penetration. Biophys. J. 82, 3289-3304. doi: 10.1016/S0006-3495(02)75670-1

Freddolino, P. L., Harrison, C. B., Liu, Y., and Schulten, K. (2010). Challenges in protein folding simulations: timescale, representation, and analysis. Nat. Phys. 6, 751-758. doi: 10.1038/nphys1713

Freddolino, P. L., Park, S., Roux, B., and Schulten, K. (2009). Force field bias in protein folding simulations. Biophys. J. 96, 3772-3780. doi: 10.1016/j.bpj.2009.02.033

Friesner, R. A. (2005). "Modeling polarization in proteins and protein-ligand complexes: methods and preliminary results," in Advances in Protein Chemistry. eds R. L. Baldwin and D. Baker (Amsterdam: Academic Press), 79-104. doi: 10.1016/S0065-3233(05)72003-9

García-Moreno, B., Dwyer, J. J., Gittis, A. G., Lattman, E. E., Spencer, D. S., and Stites, W. E. (1997). Experimental measurement of the effective dielectric in the hydrophobic core of a protein. Biophys. Chem. 64, 211-224. doi: 10.1016/S0301-4622(96)02238-7

Gresh, N., Cisneros, G. A., Darden, T. A., and Piquemal, J.-P. (2007). Anisotropic, polarizable molecular mechanics studies of inter- and intramolecular interactions and ligand-macromolecule complexes. A Bottom-Up Strategy. J. Chem. Theory Comput. 3, 1960-1986. doi: 10.1021/ct $700134 \mathrm{r}$

Gresh, N., Perahia, D., de Courcy, B., Foret, J., Roux, C., El-Khoury, L., et al. (2016). Complexes of a $\mathrm{Zn}$-metalloenzyme binding site with hydroxamatecontaining ligands. A case for detailed benchmarkings of polarizable molecular mechanics/dynamics potentials when the experimental binding structure is unknown. J. Comput. Chem. 37, 2770-2782. doi: 10.1002/jcc.24503
Gresh, N., Piquemal, J.-P., and Krauss, M. (2005). Representation of $\mathrm{Zn}(\mathrm{II})$ complexes in polarizable molecular mechanics. Further refinements of the electrostatic and short-range contributions. Comparisons with parallel $a b$ initio computations. J. Comput. Chem. 26, 1113-1130. doi: 10.1002/jcc.20244

Harayama, T., and Riezman, H. (2018). Understanding the diversity of membrane lipid composition. Nat. Rev. Mol. Cell Biol. 19, 281-296. doi: $10.1038 / \mathrm{nrm} .2017 .138$

Harder, E., Mackerell, A. D. Jr., and Roux, B. (2009). Many-body polarization effects and the membrane dipole potential. J. Am. Chem. Soc. 131, 2760-2761. doi: $10.1021 /$ ja $806825 \mathrm{~g}$

Harger, M., Li, D., Wang, Z., Dalby, K., Lagardère, L., Piquemal, J.-P., et al. (2017). Tinker-OpenMM: absolute and relative alchemical free energies using AMOEBA on GPUs. J. Comput. Chem. 38, 2047-2055. doi: 10.1002/jcc.24853

Hauser, H., Hinckley, C. C., Krebs, J., Levine, B. A., Phillips, M. C., and Williams, R. J. (1977). The interaction of ions with phosphatidylcholine. Biochim. Biophys. Acta 468, 364-377. doi: 10.1016/0005-2736(77)90288-7

Huang, J., Lemkul, J. A., Eastman, P. K., and MacKerell, A. D. Jr. (2018). Molecular dynamics simulations using the drude polarizable force field on GPUs with OpenMM: Implementation, validation, and benchmarks. J. Comput. Chem. 39, 1682-1689. doi: 10.1002/jcc.25339

Huang, J., and MacKerell, A. D. Jr. (2013). CHARMM36 all-atom additive protein force field: validation based on comparison to NMR data. J. Comput. Chem. 34, 2135-2145. doi: 10.1002/jcc.23354

Huang, J., and MacKerell, A. D. Jr. (2014). Induction of peptide bond dipoles drives cooperative helix formation in the (AAQAA)3 peptide. Biophys. J. 107, 991-997. doi: 10.1016/j.bpj.2014.06.038

Huang, J., Simmonett, A. C., Pickard, F. C. IV., MacKerell, A. D. Jr., and Brooks, B. R. (2017). Mapping the drude polarizable force field onto a multipole and induced dipole model. J. Chem. Phys. 147:161702. doi: 10.1063/ 1.4984113

Javanainen, M., Melcrová, A. A., Magarkar, A., Jurkiewicz, P., Hof, M., Jungwirth, P., et al. (2017). Two cations, two mechanisms: interactions of sodium and calcium with zwitterionic lipid membranes. Chem. Commun. 53, 5380-5383. doi: 10.1039/C7CC02208E

Jiang, W., Hardy, D. J., Phillips, J. C., Mackerell, A. D. Jr., Schulten, K., and Roux, B. (2011). High-performance scalable molecular dynamics simulations of a polarizable force field based on classical Drude oscillators in NAMD. J. Phys. Chem. Lett. 2, 87-92. doi: 10.1021/jz101461d

Jiao, D., Golubkov, P. A., Darden, T. A., and Ren, P. (2008). Calculation of protein-ligand binding free energy by using a polarizable potential. Proc. Natl. Acad. Sci. U.S.A. 105, 6290-6295. doi: 10.1073/pnas.0711 686105

Jing, Z., Liu, C., Cheng, S. Y., Qi, R., Walker, B. D., Piquemal, J.P., et al. (2019). Polarizable force fields for biomolecular simulations: recent advances and applications. Annu. Rev. Biophys. 48, 371-394. doi: 10.1146/annurev-biophys-070317-033349

Jing, Z., Liu, C., Qi, R., and Ren, P. (2018). Many-body effect determines the selectivity for $\mathrm{Ca}$ and $\mathrm{Mg}$ in proteins. Proc. Natl. Acad. Sci. U.S.A. 115, E7495-E7501. doi: 10.1073/pnas.1805049115

Jolly, L. H., Duran, A., Lagardère, L., Ponder, J. W., Ren, P. Y., and Piquemal, J.-P. (2019). Raising the performance of the Tinker-HP molecular modeling package [article v1.0]. LiveCoMS 1:10409. doi: 10.33011/livecoms.1.2.10409

Jorgensen, W. L. (2007). Special issue on polarization. J. Chem. Theory Comput. 3:1877. doi: $10.1021 /$ ct700252g

Kleshchonok, A., and Tkatchenko, A. (2018). Tailoring van der Waals dispersion interactions with external electric charges. Nat. Commun. 9:3017. doi: 10.1038/s41467-018-05407-x

Kohagen, M., Mason, P. E., and Jungwirth, P. (2014). Accurate description of calcium solvation in concentrated aqueous solutions. J. Phys. Chem. B 118, 1-27. doi: 10.1021/jp5005693

Kohagen, M., Pluharová, E., Mason, P. E., and Jungwirth, P. (2015). Exploring ion-ion interactions in aqueous solutions by a combination of molecular dynamics and neutron scattering. J. Phys. Chem. Lett. 6, 1563-1567. doi: 10.1021/acs.jpclett.5b00060

Kurland, R., Newton, C., Nir, S., and Papahadjopoulos, D. (1979). Specificity of $\mathrm{Na}+$ binding to phosphatidylserine vesicles from a $23 \mathrm{Na}$ NMR relaxation rate study. Biochim. Biophys. Acta 551, 137-147. doi: 10.1016/0005-2736(79)90360-2 
Lagardère, L., Aviat, F., and Piquemal, J.-P. (2019). Pushing the limits of multipletime-step strategies for polarizable point dipole molecular dynamics. J. Phys. Chem. Lett. 10, 2593-2599. doi: 10.1021/acs.jpclett.9b00901

Lagardère, L., Jolly, L.-H., Lipparini, F., Aviat, F., Stamm, B., Jing, Z. F., et al. (2018). Tinker-HP: a massively parallel molecular dynamics package for multiscale simulations of large complex systems with advanced point dipole polarizable force fields. Chem. Sci. 9, 956-972. doi: 10.1039/C7SC04531J

Lagardère, L., Lipparini, F., Polack, É., Stamm, B., Cancès, É., Schnieders, M., et al. (2015). Scalable evaluation of polarization energy and associated forces in polarizable molecular dynamics: II. Toward massively parallel computations using smooth particle mesh Ewald. J. Chem. Theory Comput. 11, 2589-2599. doi: $10.1021 /$ acs.jctc.5b00171

Lamoureux, G., MacKerell, A. D. Jr., and Roux, B. (2003). A simple polarizable model of water based on classical Drude oscillators. J. Chem. Phys. 119, 5185-5197. doi: 10.1063/1.1598191

Lee, A. G. (2004). How lipids affect the activities of integral membrane proteins. Biochim. Biophys. Acta 1666, 62-87. doi: 10.1016/j.bbamem.2004.05.012

Lee, S., and Park, S. S. (2011). Dielectric properties of organic solvents from non-polarizable molecular dynamics simulation with electronic continuum model and density functional theory. J. Phys. Chem. B 115, 12571-12576. doi: $10.1021 /$ jp207658m

Lemkul, J. A., Huang, J., Roux, B., and Mackerell, A. D. (2016). An empirical polarizable force field based on the classical drude oscillator model: development history and recent applications. Chem. Rev. 116, 4983-5013. doi: $10.1021 /$ acs.chemrev.5b00505

Leontyev, I., and Stuchebrukhov, A. (2011). Accounting for electronic polarization in non-polarizable force fields. Phys. Chem. Chem. Phys. 13:2613. doi: $10.1039 / \mathrm{c} 0 \mathrm{cp} 01971 \mathrm{~b}$

Leontyev, I. V., and Stuchebrukhov, A. A. (2009). Electronic continuum model for molecular dynamics simulations. J. Chem. Phys. 130:085102. doi: $10.1063 / 1.3060164$

Leontyev, I. V., and Stuchebrukhov, A. A. (2010a). Electronic continuum model for molecular dynamics simulations of biological molecules. J. Chem. Theory Comput. 6, 1498-1508. doi: 10.1021/ct9005807

Leontyev, I. V., and Stuchebrukhov, A. A. (2010b). Electronic polarizability and the effective pair potentials of water. J. Chem. Theory Comput. 6, 3153-3161. doi: $10.1021 / \mathrm{ct} 1002048$

Leontyev, I. V., and Stuchebrukhov, A. A. (2012). Polarizable mean-field model of water for biological simulations with amber and charmm force fields. J. Chem. Theory Comput. 8, 3207-3216. doi: 10.1021/ct300011h

Lipparini, F., Lagardère, L., Stamm, B., Cancès, E., Schnieders, M., Ren, P., et al. (2014). Scalable Evaluation of Polarization Energy and Associated Forces in Polarizable Molecular Dynamics: I. Toward Massively Parallel Direct Space Computations. J. Chem. Theory Comput. 10, 1638-1651. doi: 10.1021/ ct401096t

Liu, C., Piquemal, J.-P., and Ren, P. (2019). AMOEBA+ classical potential for modeling molecular interactions. J. Chem. Theory Comput. 15, 4122-4139. doi: $10.1021 /$ acs.jctc. 9 b00261

Loco, D., Lagardère, L., Caprasecca, S., Lipparini, F., Mennucci, B., and Piquemal, J.-P. (2017). Hybrid QM/MM molecular dynamics with AMOEBA polarizable embedding. J. Chem. Theory Comput. 13, 4025-4033. doi: 10.1021/acs.jctc.7b00572

Loco, D., Lagardère, L., Cisneros, G. A., Scalmani, G., Frisch, M., Lipparini, F., et al. (2019). Towards large scale hybrid QM/MM dynamics of complex systems with advanced point dipole polarizable embeddings. Chem. Sci. 10, 7200-7211. doi: 10.1039/C9SC01745C

Loco, D., Polack, É., Caprasecca, S., Lagardère, L., Lipparini, F., Piquemal, J.-P., et al. (2016). A QM/MM approach using the AMOEBA polarizable embedding: from ground state energies to electronic excitations. J. Chem. Theory Comput. 12, 3654-3661. doi: 10.1021/acs.jctc.6b00385

Lopes, P. E. M., Guvench, O., and MacKerell, A. D. Jr. (2015). Current status of protein force fields for molecular dynamics simulations. Methods Mol. Biol. 1215, 47-71. doi: 10.1007/978-1-4939-1465-4_3

Lopes, P. E. M., Huang, J., Shim, J., Luo, Y., Li, H., Roux, B., et al. (2013). Polarizable force field for peptides and proteins based on the classical drude oscillator. J. Chem. Theory Comput. 9, 5430-5449. doi: 10.1021/ct40 0781b
Lopes, P. E. M., Roux, B., and Mackerell, A. D. Jr. (2009). Molecular modeling and dynamics studies with explicit inclusion of electronic polarizability. Theory and applications. Theor. Chem. Acc. 124, 11-28. doi: 10.1007/s00214-009-0617-x

Lucas, T. R., Bauer, B. A., and Patel, S. (2012). Charge equilibration force fields for molecular dynamics simulations of lipids, bilayers, and integral membrane protein systems. BBA - Biomembranes 1818, 318-329. doi: 10.1016/j.bbamem.2011.09.016

Lund, M., Vacha, R., and Jungwirth, P. (2008). Specific ion binding to macromolecules: effects of hydrophobicity and ion pairing. Langmuir 24, 3387-3391. doi: 10.1021/la7034104

Magarkar, A., Jurkiewicz, P., Allolio, C., Hof, M., and Jungwirth, P. (2017). Increased binding of calcium ions at positively curved phospholipid membranes. J. Phys. Chem. Lett. 8, 518-523. doi: 10.1021/acs.jpclett.6b02818

Maier, J. A., Martinez, C., Kasavajhala, K., Wickstrom, L., Hauser, K. E., and Simmerling, C. (2015). ff14SB: improving the accuracy of protein side chain and backbone parameters from ff99SB. J. Chem. Theory Comput. 11, 3696-3713. doi: $10.1021 /$ acs.jctc.5b00255

Martinek, T., Duboué-Dijon, E., Timr, Š., Mason, P. E., Baxová, K., Fischer, H. E., et al. (2018). Calcium ions in aqueous solutions: Accurate force field description aided by ab initio molecular dynamics and neutron scattering. J. Chem. Phys. 148:222813. doi: 10.1063/1.5006779

Mason, P. E., Jungwirth, P., and Duboué-Dijon, E. (2019). Quantifying the strength of a salt bridge by neutron scattering and molecular dynamics. J. Phys. Chem. Lett. 10, 3254-3259. doi: 10.1021/acs.jpclett.9b01309

Mason, P. E., Wernersson, E., and Jungwirth, P. (2012). Accurate description of aqueous carbonate ions: an effective polarization model verified by neutron scattering. J. Phys. Chem. B 116, 8145-8153. doi: 10.1021/jp3008267

Mattai, J., Hauser, H., Demel, R. A., and Shipley, G. G. (1989). Interactions of metal ions with phosphatidylserine bilayer membranes: effect of hydrocarbon chain unsaturation. Biochemistry 28, 2322-2330. doi: 10.1021/bi00431a051

Melcr, J., Ferreira, T., Jungwirth, P., and Ollila, O. H. S. (2019). Improved Cation Binding to Lipid Bilayer With Negatively Charged POPS by Effective Inclusion of Electronic Polarization. Preprint manuscript at Zenodo.org.

Melcr, J., Martinez-Seara, H., Nencini, R., Kolafa, J., Jungwirth, P., and Ollila, O. H. S. (2018). Accurate binding of sodium and calcium to a POPC bilayer by effective inclusion of electronic polarization. J. Phys. Chem. B 122, 4546-4557. doi: $10.1021 /$ acs.jpcb.7b12510

Melcrová, A., Pokorná, S., Pullanchery, S., Kohagen, M., Jurkiewicz, P., Hof, M., et al. (2016). The complex nature of calcium cation interactions with phospholipid bilayers. Sci. Rep. 6:38035. doi: 10.1038/srep38035

Morozov, A. V., Tsemekhman, K., and Baker, D. (2006). Electron density redistribution accounts for half the cooperativity of alpha helix formation. J. Phys. Chem. B 110, 4503-4505. doi: 10.1021/jp057161f

Mu, X., Wang, Q., Wang, L.-P., Fried, S. D., Piquemal, J.-P., Dalby, K. N., et al. (2014). Modeling organochlorine compounds and the $\sigma$-hole effect using a polarizable multipole force field. J. Phys. Chem. B 118, 6456-6465. doi: $10.1021 /$ jp411671a

Narth, C., Lagardère, L., Polack, É., Gresh, N., Wang, Q., Bell, D. R., et al. (2016). Scalable improvement of SPME multipolar electrostatics in anisotropic polarizable molecular mechanics using a general short-range penetration correction up to quadrupoles. J. Comput. Chem. 37, 494-506. doi: $10.1002 /$ jcc. 24257

Patel, S., Davis, J. E., and Bauer, B. A. (2009). Exploring ion permeation energetics in gramicidin A using polarizable charge equilibration force fields. J. Am. Chem. Soc. 131, 13890-13891. doi: 10.1021/ja90 $2903 \mathrm{~m}$

Pegado, L., Marsalek, O., Jungwirth, P., and Wernersson, E. (2012). Solvation and ion-pairing properties of the aqueous sulfate anion: explicit versus effective electronic polarization. Phys. Chem. Chem. Phys. 14, 10248-10257. doi: $10.1039 / \mathrm{c} 2 \mathrm{cp} 40711 \mathrm{f}$

Phillips, J. C., Zheng, G., Kumar, S., and Kale, L. V. (2002). NAMD: biomolecular simulation on thousands of processors. In: ACM/IEEE SC 2002 Conference (SC'02) (Baltimore, MD). doi: 10.1109/SC.2002.10019

Piana, S., Klepeis, J. L., and Shaw, D. E. (2014). Assessing the accuracy of physical models used in protein-folding simulations: quantitative evidence from long molecular dynamics simulations. Curr. Opin. Struct. Biol. 24, 98-105. doi: $10.1016 /$ j.sbi.2013.12.006 
Piana, S., Lindorff-Larsen, K., and Shaw, D. E. (2011). How robust are protein folding simulations with respect to force field parameterization? Biophys. J. 100, L47-L49. doi: 10.1016/j.bpj.2011.03.051

Piquemal, J.-P., Chevreau, H., and Gresh, N. (2007). Toward a separate reproduction of the contributions to the hartree-fock and DFT intermolecular interaction energies by polarizable molecular mechanics with the SIBFA potential. J. Chem. Theory Comput. 3, 824-837. doi: 10.1021/ct7000182

Piquemal, J.-P., and Cisneros, G. (2016). "Status of the gaussian electrostatic model, a density-based polarizable force field," in Many-Body Effects and Electrostatics in Biomolecules, eds Q. Cui, P. Ren, and M. Meuwly (Pan Stanford Publishing), 269-299. doi: 10.1201/b21343-11

Piquemal, J.-P., Cisneros, G. A., Reinhardt, P., Gresh, N., and Darden, T. A. (2006a). Towards a force field based on density fitting. J. Chem. Phys. 124:104101. doi: 10.1063/1.2173256

Piquemal, J.-P., Gresh, N., and Giessner-Prettre, C. (2003). Improved formulas for the calculation of the electrostatic contribution to the intermolecular interaction energy from multipolar expansion of the electronic distribution. J. Phys. Chem. A 107, 10353-10359. doi: 10.1021/jp035748t

Piquemal, J.-P., and Jordan, K. D. (2017). Preface: special topic: from quantum mechanics to force fields. J. Chem. Phys. 147:161401. doi: 10.1063/1.5008887

Piquemal, J.-P., Perera, L., Cisneros, G. A., Ren, P., Pedersen, L. G., and Darden, T. A. (2006b). Towards accurate solvation dynamics of divalent cations in water using the polarizable amoeba force field: From energetics to structure. J. Chem. Phys. 125:054511. doi: 10.1063/1.2234774

Pluhackova, K., Kirsch, S. A., Han, J., Sun, L., Jiang, Z., Unruh, T., et al. (2016). A critical comparison of biomembrane force fields: structure and dynamics of model DMPC, POPC, and POPE bilayers. J. Phys. Chem. B 120, 3888-3903. doi: 10.1021/acs.jpcb.6b01870

Pluhařová, E., Mason, P. E., and Jungwirth, P. (2013). Ion pairing in aqueous lithium salt solutions with monovalent and divalent counter-anions. J. Phys. Chem. A 117, 11766-11773. doi: 10.1021/jp402532e

Ponder, J. W., and Case, D. A. (2003). Force fields for protein simulations. Adv. Protein Chem. 66, 27-85. doi: 10.1016/S0065-3233(03)66002-X

Qi, R., Jing, Z., Liu, C., Piquemal, J.-P., Dalby, K. N., and Ren, P. (2018). Elucidating the phosphate binding mode of phosphate-binding protein: the critical effect of buffer solution. J. Phys. Chem. B 122, 6371-6376. doi: 10.1021/acs.jpcb. 8 b03194

Rackers, J. A., and Ponder, J. W. (2019). Classical Pauli repulsion: An anisotropic, atomic multipole model. J. Chem. Phys. 150:084104. doi: 10.1063/ 1.5081060

Rackers, J. A., Wang, Z., Lu, C., Laury, M. L., Lagardère, L., Schnieders, M. J., et al. (2018). Tinker 8: software tools for molecular design. J. Chem. Theory Comput. 14, 5273-5289. doi: 10.1021/acs.jctc.8b00529

Ren, P., and Ponder, J. W. (2003). Polarizable atomic multipole water model for molecular mechanics simulation. J. Phys. Chem. B 107, 5933-5947. doi: $10.1021 /$ jp027815+

Rick, S. W., and Stuart, S. J. (2003). "Potentials and algorithms for incorporating polarizability in computer simulations," in Reviews in Computational Chemistry, eds K. B. Lipkowitz and D. B. Boyd (Hoboken, NJ: John Wiley \& Sons) 89-146

Riera, M., Lambros, E., Nguyen, T. T., Götz, A. W., and Paesani, F. (2019). Loworder many-body interactions determine the local structure of liquid water. Chem. Sci. 116:7463. doi: 10.1039/C9SC03291F

Robertson, M. J., Tirado-Rives, J., and Jorgensen, W. L. (2015). Improved peptide and protein torsional energetics with the OPLSAA force field. J. Chem. Theory Comput. 11, 3499-3509. doi: 10.1021/acs.jctc.5b00356

Roux, M., and Bloom, M. (1990). Calcium, magnesium, lithium, sodium, and potassium distributions in the headgroup region of binary membranes of phosphatidylcholine and phosphatidylserine as seen by deuterium NMR. Biochemistry 29, 7077-7089. doi: 10.1021/bi00482a019
Roux, M., and Bloom, M. (1991). Calcium binding by phosphatidylserine headgroups. Deuterium NMR study. Biophys. J. 60, 38-44. doi: 10.1016/S0006-3495(91)82028-8

Schröder, C. (2012). Comparing reduced partial charge models with polarizable simulations of ionic liquids. Phys. Chem. Chem. Phys. 14, 3089-3102. doi: $10.1039 / \mathrm{c} 2 \mathrm{cp} 23329 \mathrm{k}$

Shi, Y., Jiao, D., Schnieders, M. J., and Ren, P. (2009). Trypsin-ligand binding free energy calculation with AMOEBA. Conf. Proc. IEEE Eng. Med. Biol. Soc. 2009, 2328-2331. doi: 10.1109/IEMBS.2009.5335108

Shi, Y., Ren, P., Schnieders, M., and Piquemal, J.-P. (2015). "Polarizable force fields for biomolecular modeling: parrill/reviews in computational chemistry volume 28," in Reviews in Computational Chemistry Volume 28 Reviews in Computational Chemistry, eds A. L. Parrill and K. B. Lipkowitz (Hoboken, NJ: John Wiley and Sons, Inc.), 51-86. doi: 10.1002/9781118889886.ch2

Shi, Y., Xia, Z., Zhang, J., Best, R., Wu, C., Ponder, J. W., et al. (2013). The Polarizable atomic multipole-based AMOEBA force field for proteins. J. Chem. Theory Comput. 9, 4046-4063. doi: 10.1021/ct4003702

Stone, A. (2013). The Theory of Intermolecular Forces. Oxford. doi: 10.1093/acprof:oso/9780199672394.001.0001

Vacha, R., Siu, S. W. I., Petrov, M., Böckmann, R. A., Barucha-Kraszewska, J. J., Jurkiewicz, P., et al. (2009). Effects of alkali cations and halide anions on the DOPC lipid membrane. J. Phys. Chem. A 113, 7235-7243. doi: $10.1021 /$ jp809974e

Van Der Spoel, D., Lindahl, E., Hess, B., Groenhof, G., Mark, A. E., and Berendsen, H. J. C. (2005). GROMACS: fast, flexible, and free. J. Comput. Chem. 26, 1701-1718. doi: 10.1002/jcc.20291

Vazdar, M., Jungwirth, P., and Mason, P. E. (2013). Aqueous guanidiniumcarbonate interactions by molecular dynamics and neutron scattering: relevance to ion-protein interactions. J. Phys. Chem. B 117, 1844-1848. doi: $10.1021 /$ jp310719g

Venable, R. M., Krämer, A., and Pastor, R. W. (2019). Molecular dynamics simulations of membrane permeability. Chem. Rev. 119, 5954-5997. doi: 10.1021/acs.chemrev.8b00486

Wang, Q., Rackers, J. A., He, C., Qi, R., Narth, C., Lagardère, L., et al. (2015). General model for treating short-range electrostatic penetration in a molecular mechanics force field. J. Chem. Theory Comput. 11, 2609-2618. doi: 10.1021 /acs.jctc. 5 b00267

Wang, W., and Skeel, R. D. (2005). Fast evaluation of polarizable forces. J. Chem. Phys. 123:164107. doi: 10.1063/1.2056544

Wu, J. C., Piquemal, J.-P., Chaudret, R., Reinhardt, P., and Ren, P. (2010). Polarizable molecular dynamics simulation of $\mathrm{Zn}(\mathrm{II})$ in water using the AMOEBA force field. J. Chem. Theory Comput. 6, 2059-2070. doi: $10.1021 /$ ct100091j

Zhang, C., Lu, C., Jing, Z., Wu, C., Piquemal, J.-P., Ponder, J. W., et al. (2018). AMOEBA polarizable atomic multipole force field for nucleic acids. J. Chem. Theory Comput. 14, 2084-2108. doi: 10.1021/acs.jctc.7b01169

Zhang, J., Yang, W., Piquemal, J.-P., and Ren, P. (2012). Modeling structural coordination and ligand binding in zinc proteins with a polarizable potential. J. Chem. Theory Comput. 8, 1314-1324. doi: 10.1021/ct200812y

Conflict of Interest: The authors declare that the research was conducted in the absence of any commercial or financial relationships that could be construed as a potential conflict of interest.

Copyright (c) 2019 Melcr and Piquemal. This is an open-access article distributed under the terms of the Creative Commons Attribution License (CC BY). The use, distribution or reproduction in other forums is permitted, provided the original author(s) and the copyright owner(s) are credited and that the original publication in this journal is cited, in accordance with accepted academic practice. No use, distribution or reproduction is permitted which does not comply with these terms. 